\title{
Analytic Solutions for Heat Conduction in Functionally Graded Circular Hollow Cylinders with Time-Dependent Boundary Conditions
}

\author{
Sen-Yung Lee and Chih-Cheng Huang \\ Department of Mechanical Engineering, National Cheng Kung University, Tainan 701, Taiwan \\ Correspondence should be addressed to Sen-Yung Lee; sylee@mail.ncku.edu.tw
}

Received 16 May 2013; Revised 27 June 2013; Accepted 1 July 2013

Academic Editor: Abdelouahed Tounsi

Copyright (C) 2013 S.-Y. Lee and C.-C. Huang. This is an open access article distributed under the Creative Commons Attribution License, which permits unrestricted use, distribution, and reproduction in any medium, provided the original work is properly cited.

An analytic solution method, without integral transformation, is developed to find the exact solutions for transient heat conduction in functionally graded (FG) circular hollow cylinders with time-dependent boundary conditions. By introducing suitable shifting functions, the governing second-order regular singular differential equation with variable coefficients and time-dependent boundary conditions is transformed into a differential equation with homogenous boundary conditions. The exact solution of the system with thermal conductivity and specific heat in power functions with different orders is developed. Finally, limiting studies and numerical analyses are given to illustrate the efficiency and the accuracy of the analysis.

\section{Introduction}

The applications of heat conduction in functionally graded (FG) circular hollow cylinders with time-dependent boundary conditions can be widely found in many engineering fields, such as cannon barrels, heat exchanger tubes, time varied heating on walls of circular structure, and heat treatment on hollow cylinders. As such, an accurate solution method is very helpful for relevant developments.

The problem of heat conduction with time-dependent boundary conditions cannot be solved directly by the method of separation of variables. In most of the analyses, an integral transform has been used to remove the time-dependent term. For the problem of heat conduction in circular hollow uniform cylinders with time-dependent boundary conditions, the associated governing differential equation is a secondorder Bessel differential equation with constant coefficients. After conducting a Laplace transformation, the analytical solution can be obtained and found in the book by Özisik [1].

When the structure is an FG circular hollow cylinder, the associated governing differential equation is a secondorder regular singular differential equation with variable coefficients. For problems with time-independent boundary conditions, various numerical methods have been developed, such as the perturbation method [2], the finite difference method [3], and the finite element method [4]. Jabbari et al. $[5,6]$ derived analytical solutions for thermal stresses of FG hollow cylinders whose material properties vary with power law distribution through the thickness due to radially symmetric loads and nonaxisymmetric loads. By using the Laplace transformation and a series expansion of Bessel functions, Ootao and Tanigawa [7] analyzed one-dimensional transient thermoelastic problem with the material properties varying with the powerlaw form of the radial coordinate variable. Zhao et al. [8] analyzed the temperature change when the thermal and thermoelastic properties are assumed to vary exponentially in the radial direction. Hosseini et al. [9] considered the material properties to be nonlinear with a power law distribution through the thickness, while the temperature distribution was derived analytically using the Bessel functions.

In the study of heat conduction in FG circular hollow cylinders with time-dependent boundary conditions, only limited studies can be found. Ootao et al. [10] studied the transient temperature and thermal stress distribution in an infinitely long nonhomogeneous hollow cylinder due to 
a moving heat source in the axial direction from the inner and/or outer surfaces using the layerwise theory in conjunction with the method of Fourier cosine and the Laplace transformations. Shao and Ma [11] employed the Laplace transform techniques and the series solving method to study thermomechanical stresses in FG circular hollow cylinders with linearly increasing boundary temperature. Jabbari et al. [12] developed an analytical solution for the one-dimensional temperature distribution, mechanical and thermal stresses in an infinitely long FG hollow cylinder under a moving heat source, which moves across the thickness of the cylinder. Asgari and Akhlaghi [13] employed the finite element method to study the transient thermal stresses in a thick hollow cylinder with finite length mad of 2D-FGM that its material properties are varied in the radial and axial directions with a power law function. The thermal boundary conditions at the inner and outer radiuses are time dependent. Singh et al. [14] applied the finite integral transform method and the separation of variables method to solve time-dependent heat conduction problem in a multilayer annulus. Malekzadeh and Heydarpour [15] used the differential quadrature method (DQM) in conjunction with the finite element method (FEM) to study the response of FG cylindrical shells under moving thermomechanical loads. Recently, Wang and Liu [16] have employed the method of separation of variables to develop the analytical solution of transient temperature fields for twodimensional transient heat conduction in a fiber-reinforced multilayer cylindrical composite.

In the study of thermal elastic response of FG cylinders without mechanical loading, the heat conduction problem is not incorporated with the elastic field and can be studied independently. However, the thermal field will be coupled with the temperature field. In this paper, one considers the heat conduction problem of FG cylinders only. A new solution method, which is a modification on the method developed by Lee and Lin [17] and Chen et al. [18], is used to develop the analytical solution for transient heat conduction in FG circular hollow cylinders with time-dependent boundary conditions. By introducing suitable shifting functions, the governing second-order differential equation with variable coefficients and time-dependent boundary conditions is transformed into a differential equation with homogenous boundary conditions. The analytic solution of the system with thermal conductivity and specific heat in power functions with different orders is developed. Finally, limiting studies and numerical analysis are given to illustrate the efficiency and accuracy of the solution method.

\section{Mathematical Modeling}

Consider the transient heat conduction in an FG circular hollow cylinder with time-dependent boundary condition at the inner and outer surfaces, as shown in Figure 1. The governing differential equation of the system is

$$
\begin{array}{r}
\frac{1}{r} \frac{1}{\partial r}\left[r k(r) \frac{\partial T(r, t)}{\partial r}\right]+\dot{g}(r, t)=\rho c(r) \frac{\partial T(r, t)}{\partial t} . \\
a<r<b, t>0 .
\end{array}
$$

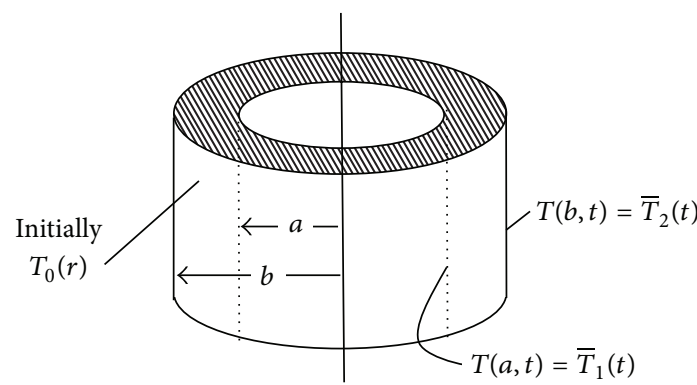

FIGURE 1: FG circular hollow cylinder with time-dependent boundary conditions.

The boundary conditions are

$$
\begin{aligned}
& T(a, t)=\bar{T}_{1}(t), \\
& T(b, t)=\bar{T}_{2}(t),
\end{aligned}
$$

and the initial condition is

$$
T(r, 0)=T_{0}(r)
$$

Here, $r$ is the space variable and $t$ is the time variable, $c(r)$ is the specific heat, $k(r)$ is the thermal conductivity, $T(r, t)$ is the temperature, $\rho$ is the mass density, and $\dot{g}(r, t)$ is the heat source inside the circular hollow cylinder. $\bar{T}_{1}(t)$ and $\bar{T}_{2}(t)$ are the time-dependent temperatures at the inner and outer surfaces, respectively.

In terms of the following dimensionless parameters

$$
\begin{gathered}
K(\xi)=\frac{k(r)}{k(a)}, \quad C(\xi)=\frac{c(r)}{c(a)}, \quad G(\xi, \tau)=\frac{\dot{g}(r, t) b^{2}}{k(a) T_{r}}, \\
\xi=\frac{r}{b}, \quad \bar{r}=\frac{a}{b}, \quad \theta=\frac{T}{T_{r}}, \quad \theta_{0}=\frac{T_{0}}{T_{r}}, \\
\bar{\theta}_{i}=\frac{\bar{T}_{i}}{T_{r}}, \quad \tau=\frac{k(a) t}{c(a) \rho b^{2}},
\end{gathered}
$$

where $T_{r}$ is the reference temperature, the boundary value problem of heat conduction becomes

$$
\begin{gathered}
\frac{1}{\xi} \frac{1}{\partial \xi}\left[\xi K(\xi) \frac{\partial \theta(\xi, \tau)}{\partial \xi}\right]+G(\xi, \tau)=C(\xi) \frac{\partial \theta(\xi, \tau)}{\partial \tau}, \\
\bar{r}<\xi<1, \tau>0, \\
\theta(\bar{r}, \tau)-\bar{\theta}_{1}=0, \\
\theta(1, \tau)-\bar{\theta}_{2}=0, \\
\theta(\xi, 0)=\theta_{0}(\xi) .
\end{gathered}
$$




\section{Solution Method}

3.1. Change of Variables. To find the solution for the secondorder differential equation with nonhomogeneous boundary conditions, the shifting variable method developed by Lee and Lin [17] and Chen et al. [18] was extended by taking

$$
\theta(\xi, \tau)=\nu(\xi, \tau)+f_{1}(\tau) g_{1}(\xi)+f_{2}(\tau) g_{2}(\xi),
$$

where $g_{i}(\xi), i=1,2$ are shifting functions to be specified and $\nu(\xi, \tau)$ is the transformed function. Substituting (6) into (5) yields the following partial differential equation:

$$
\begin{aligned}
\frac{1}{\xi} \frac{\partial}{\partial \xi}\left[\xi K(\xi) \frac{\partial \nu(\xi, \tau)}{\partial \xi}\right]-C(\xi) \frac{\partial \nu(\xi, \tau)}{\partial \tau} \\
\quad=-G(\xi, \tau)+\sum_{i=1}^{2}\left\{\begin{array}{c}
C(\xi) g_{i}(\xi) \frac{d f_{i}(\tau)}{d \tau} \\
\left.-f_{i}(\tau) \frac{1}{\xi} \frac{d}{d \xi}\left[\xi K(\xi) \frac{d g_{i}(\xi)}{d \xi}\right]\right\},
\end{array}\right.
\end{aligned}
$$

the associated boundary conditions:

$$
\begin{aligned}
& \nu(\bar{r}, \tau)+f_{1}(\tau) g_{1}(\bar{r})+f_{2}(\tau) g_{2}(\bar{r})=f_{1}(\tau), \\
& \nu(1, \tau)+f_{1}(\tau) g_{1}(1)+f_{2}(\tau) g_{2}(1)=f_{2}(\tau),
\end{aligned}
$$

and the associated initial condition

$$
\nu(\xi, 0)=\theta(\xi, 0)-f_{1}(0) g_{1}(\xi)-f_{2}(0) g_{2}(\xi)
$$

3.2. Shifting Functions. To simplify the analysis, the shifting functions are specifically chosen such that they satisfy the following differential equations and the boundary conditions

$$
\begin{gathered}
\frac{d^{2} g_{i}(\xi)}{d \xi^{2}}=0, \quad i=1,2, \\
g_{1}(\bar{r})=1, \\
g_{1}(1)=0, \\
g_{2}(\bar{r})=0, \\
g_{2}(1)=1 .
\end{gathered}
$$

These two shifting functions can be easily determined as

$$
\begin{gathered}
g_{1}(\xi)=\frac{1}{1-\bar{r}}(1-\xi), \\
g_{2}(\xi)=\frac{1}{1-\bar{r}}(-\bar{r}+\xi) .
\end{gathered}
$$

3.3. Reduced Homogenous Problem. With these two shifting functions, (11), the governing differential equation (7) becomes

$$
\frac{1}{\xi} \frac{\partial}{\partial \xi}\left[\xi K(\xi) \frac{\partial \nu(\xi, \tau)}{\partial \xi}\right]-C(\xi) \frac{\partial \nu(\xi, \tau)}{\partial \tau}=F(\xi, \tau),
$$

where

$$
\begin{aligned}
& F(\xi, \tau) \\
& =-G(\xi, \tau) \\
& \quad+\sum_{i=1}^{2}\left\{C(\xi) g_{i}(\xi) \frac{d f_{i}(\tau)}{d \tau}-\frac{f_{i}(\tau)}{\xi} \frac{d}{d \xi}[\xi K(\xi)] \frac{d g_{i}(\xi)}{d \xi}\right\}
\end{aligned}
$$

The two nonhomogenous boundary conditions, (8), for the transformed function $\nu(\xi, \tau)$ are reduced to homogenous ones:

$$
\begin{aligned}
& \nu(\bar{r}, \tau)=0, \\
& \nu(1, \tau)=0 .
\end{aligned}
$$

The transformed initial condition is

$$
\nu(\xi, 0)=\theta_{0}(\xi)-f_{1}(0) g_{1}(\xi)-f_{2}(0) g_{2}(\xi)=\nu_{0}(\xi)
$$

\subsection{Solution of Transformed Variable}

3.4.1. Characteristic Solution. To find the solution $\nu(\xi, \tau)$, we use the eigenfunction expansion method and assume the solution to be in the form

$$
\nu(\xi, \tau)=\phi(\xi) B(\tau)
$$

The separation equation for the dimensionless time variable $B(\tau)$ is

$$
\frac{d B(\tau)}{d \tau}=-\lambda^{2} B(\tau)
$$

and the dimensionless space variable $\phi(\xi)$ satisfies the following self-adjoin operator:

$$
\begin{gathered}
\frac{1}{\xi} \frac{\mathrm{d}}{d \xi}\left[\xi K(\xi) \frac{d \phi(\xi)}{d \xi}\right]+\lambda^{2} C(\xi) \phi(\xi)=0, \quad \xi \in(\bar{r}, 1) \\
\phi(\bar{r})=0 \\
\phi(1)=0 .
\end{gathered}
$$

Let $X_{j}(\xi), j=1,2$, be the two linearly independent fundamental solutions of the system; then, the solution of the differential equation (18) can be expressed as

$$
\phi(\xi)=C_{1} X_{1}(\xi)-C_{2} X_{2}(\xi)
$$

where $C_{1}$ and $C_{2}$ are constants to be determined from the boundary conditions, (19)-(20).

After substituting solutions, (21), into the boundary conditions, (19)-(20), we obtain the following characteristic equation

$$
X_{1}(1) X_{2}(\bar{r})-X_{1}(\bar{r}) X_{2}(1)=0 \text {. }
$$


Consequently, the eigenvalues $\lambda_{n}, n=1,2,3, \ldots$ can be determined. The associated $n$th eigenfunction $\phi_{n}(\xi)$ is determined as

$$
\phi_{n}(\xi)=X_{2}(1) X_{n, 1}(\xi)-X_{1}(1) X_{n, 2}(\xi)
$$

where $X_{n, 1}(\xi)$ and $X_{n, 2}(\xi)$ are, respectively, the fundamental solutions $X_{1}\left(\lambda_{n}, \xi\right)$ and $X_{2}\left(\lambda_{n}, \xi\right)$ associated with eigenvalues $\lambda_{n}, n=1,2,3, \ldots$. They are defined as $X_{n, 1}(\xi)=X_{1}\left(\lambda_{n}, \xi\right)$ and $X_{n, 2}(\xi)=X_{2}\left(\lambda_{n}, \xi\right)$. The eigenfunctions $\phi_{n}(\xi)$ constitute an orthogonal set in the interval $\bar{r} \leq \xi \leq 1$, with respect to a weighting function $\xi C(\xi)$ :

$$
\int_{\bar{r}}^{1} \xi C(\xi) \phi_{m}(\xi) \phi_{n}(\xi) d \xi= \begin{cases}0, & \text { for } m \neq n \\ \delta_{n}, & \text { for } m=n,\end{cases}
$$

where

$$
\delta_{n}=\int_{\bar{r}}^{1} \xi C(\xi) \phi_{n}^{2}(\xi) d \xi
$$

In terms of eigenfunctions, the transformed variable $\nu(\xi, \tau)$ can be expressed as

$$
\nu(\xi, \tau)=\sum_{n=1}^{\infty} \phi_{n}(\xi) B_{n}(\tau)
$$

Substituting (26) into (12), multiplying it by $\xi \phi_{m}$, and integrating from $\bar{r}$ to 1 , we obtain

$$
\frac{d B_{n}(\tau)}{d \tau}+\lambda_{n}^{2} B_{n}(\tau)=-\gamma_{n}(\tau)
$$

where

$$
\gamma_{n}(\tau)=\frac{1}{\delta_{n}} \int_{\bar{r}}^{1} \xi \phi_{n}(\xi) F(\xi, \tau) d \xi
$$

The general solution of (27) is

$$
B_{n}(\tau)=e^{-\lambda_{n}^{2} \tau}\left[\alpha_{n}-\int_{0}^{\tau} e^{\lambda_{n}^{2} \chi} \gamma_{n}(\chi) d \chi\right],
$$

$\alpha_{n}$ is determined from the initial condition (15), and

$$
\alpha_{n}=B_{n}(0)=\frac{1}{\delta_{n}} \int_{\bar{r}}^{1} \xi C(\xi) \phi_{n}(\xi) \nu_{0}(\xi) d \xi .
$$

After substituting the solution of the transformed variable (26) and the shifting functions (11) back to (6), the exact solution for the general system is obtained.

\section{Verification and Examples}

To illustrate the previous analysis, the following examples and limiting cases are given.
Example 1. Consider the heat conduction in an uniform circular hollow cylinder with time-dependent boundary conditions. The boundary value problem of the heat conduction in dimension-less form is

$$
\begin{gathered}
\frac{1}{\xi} \frac{1}{\partial \xi}\left[\xi \frac{\partial \theta(\xi, \tau)}{\partial \xi}\right]=\frac{\partial \theta(\xi, \tau)}{\partial \tau}, \quad \bar{r}<\xi<1, \tau>0, \\
\theta(\bar{r}, \tau)=\frac{\psi_{1}(\tau)}{T_{r}}, \\
\theta(1, \tau)=\frac{\psi_{2}(\tau)}{T_{r}}, \\
\theta(\xi, 0)=\frac{\bar{\theta}_{0}(\xi)}{T_{r}} .
\end{gathered}
$$

In this case, $K(\xi)=1, C(\xi)=1$. The two shifting functions are

$$
\begin{aligned}
& g_{1}(\xi)=\frac{1}{1-\bar{r}}(1-\xi), \\
& g_{2}(\xi)=\frac{1}{1-\bar{r}}(\xi-\bar{r}) .
\end{aligned}
$$

The two linearly independent fundamental solutions are

$$
\begin{aligned}
& X_{1}\left(\lambda_{n}, \xi\right)=J_{0}\left(\lambda_{n} \xi\right), \\
& X_{2}\left(\lambda_{n}, \xi\right)=Y_{0}\left(\lambda_{n} \xi\right) .
\end{aligned}
$$

This leads to

$$
\begin{gathered}
F(\xi, \tau)=\frac{1}{(1-\bar{r}) T_{r}}\left[(1-\xi) \psi_{1}^{\prime}(\tau)+(-\bar{r}+\xi) \psi_{2}^{\prime}(\tau)\right. \\
\left.+\frac{\psi_{1}(\tau)}{\xi}-\frac{\psi_{2}(\tau)}{\xi}\right]
\end{gathered}
$$

where the characteristic equation is

$$
X_{1}(1) X_{2}(\bar{r})-X_{1}(\bar{r}) X_{2}(1)=0 \text {. }
$$

The associated $n$th eigenfunction $\phi_{n}(\xi)$ is determined as

$$
\phi_{n}(\xi)=\frac{J_{0}\left(\lambda_{n} \xi\right)}{J_{0}\left(\lambda_{n}\right)}-\frac{Y_{0}\left(\lambda_{n} \xi\right)}{Y_{0}\left(\lambda_{n}\right)} .
$$

The eigenvalues $\lambda_{n}$ and the associated eigenfunctions $\phi_{n}(\xi)$ are obtained from (35) and (36). The two coefficients in (28)(29) are derived as

$$
\begin{aligned}
& \gamma_{n}(\tau)= \frac{1}{\delta_{n}} \frac{1}{(1-\bar{r}) T_{r}} \\
& \times \int_{\bar{r}}^{1}\left\{\phi _ { n } ( \xi ) \left[\left(\xi-\xi^{2}\right) \psi_{1}^{\prime}(\tau)+\left(-\bar{r} \xi+\xi^{2}\right) \psi_{2}^{\prime}(\tau)\right.\right. \\
&\left.\left.+\psi_{1}(\tau)-\psi_{2}(\tau)\right]\right\} d \xi \\
& B_{n}(\tau)=e^{-\lambda_{n}^{2} \tau}\left[\alpha_{n}-\int_{0}^{\tau} e^{\lambda_{n}^{2} \chi} \gamma_{n}(\chi) d \chi\right]
\end{aligned}
$$


where

$$
\begin{gathered}
\delta_{n}=\left[\int_{\bar{r}}^{1} \xi \phi_{n}^{2}(\xi) d \xi\right], \\
\alpha_{n}=\frac{1}{\delta_{n} T_{r}} \int_{\bar{r}}^{1} \xi \phi_{n}(\xi) \bar{\theta}_{0}(\xi) d \xi .
\end{gathered}
$$

As a result, the analytic closed solution of the system in dimensionless form is

$$
\begin{aligned}
\theta(\xi, \tau)=\sum_{n=1}^{\infty} & {\left[\phi_{n}(\xi) B_{n}(\tau)\right] } \\
& +\left(\frac{1-\xi}{1-\bar{r}}\right) \frac{\psi_{1}(\tau)}{T_{r}}+\left(\frac{-\bar{r}+\xi}{1-\bar{r}}\right) \frac{\psi_{2}(\tau)}{T_{r}},
\end{aligned}
$$

when

$$
\psi_{1}(\tau)=\psi_{2}(\tau)=0
$$

The analytic closed solution, in dimensionless form, is reduced to

$$
\theta(\xi, \tau)=\sum_{n=1}^{\infty}\left[e^{-\lambda_{n}^{2} \tau} \phi_{n}(\xi) \alpha_{n}\right]
$$

The solution is exactly the same as the one given by Özisik [1].

Example 2. Consider the heat conduction in an FG circular hollow cylinder with time-dependent boundary conditions. The coefficients of thermal conductivity and the specific heat are $K(\xi)=k_{m} \xi^{\beta_{1}}$ and $C(\xi)=c_{m} \xi^{\beta_{2}}$, respectively. The boundary value problem of the heat conduction in dimensionless form is

$$
\begin{gathered}
\frac{1}{\xi} \frac{\partial}{\partial \xi}\left[\xi K(\xi) \frac{\partial \theta(\xi, \tau)}{\partial \xi}\right]-C(\xi) \frac{\partial \theta(\xi, \tau)}{\partial \tau}=0, \\
\bar{r}<\xi<1, \tau>0, \\
\theta(\bar{r}, \tau)=0, \\
\theta(1, \tau)=\left(1-e^{-C_{0} \tau}\right) \bar{\theta}_{2}, \\
\theta(\xi, 0)=0 .
\end{gathered}
$$

In this case, two shifting functions are

$$
\begin{aligned}
& g_{1}(\xi)=\frac{1}{1-\bar{r}}(1-\xi), \\
& g_{2}(\xi)=\frac{1}{1-\bar{r}}(\xi-\bar{r}) .
\end{aligned}
$$

The route to two independent fundamental solutions of of (42) lies in the use of the Frobenius method which can be represented in terms of the Bessel functions:

$$
\begin{aligned}
& X_{1}\left(\lambda_{n}, \xi\right)=\xi^{-\beta_{1} / 2} J_{\nu}\left(\eta_{n} \xi^{\left(2+\beta_{2}-\beta_{1}\right) / 2}\right), \\
& X_{2}\left(\lambda_{n}, \xi\right)=\xi^{-\beta_{1} / 2} Y_{\nu}\left(\eta_{n} \xi^{\left(2+\beta_{2}-\beta_{1}\right) / 2}\right),
\end{aligned}
$$

where

$$
\begin{gathered}
\eta_{n}=\sqrt{\frac{c_{m}}{k_{m}}}\left(\frac{2 \lambda_{n}}{\beta_{1}-\beta_{2}-2}\right), \\
v=\frac{\beta_{1}}{\beta_{1}-\beta_{2}-2} .
\end{gathered}
$$

Now,

$F(\xi, \tau)$

$$
\begin{aligned}
= & e^{-C_{0} \tau} \bar{\theta}_{2}\left[c_{m} \xi^{\beta_{2}} C_{0}\left(a^{*}+b^{*} \xi\right)+b^{*}\left(\beta_{1}+1\right) k_{m} \xi^{\beta_{1}-1}\right] \\
& -b^{*}\left[\bar{\theta}_{2}\left(\beta_{1}+1\right) k_{m} \xi^{\beta_{1}-1}\right],
\end{aligned}
$$

where

$$
\begin{aligned}
a^{*} & =\frac{-\bar{r}}{1-\bar{r}}, \\
b^{*} & =\frac{1}{1-\bar{r}} .
\end{aligned}
$$

The characteristic equation is

$$
X_{1}(1) X_{2}(\bar{r})-X_{1}(\bar{r}) X_{2}(1)=0 .
$$

The associated $n$th eigenfunction $\phi_{n}(\xi)$ is determined as

$$
\phi_{n}(\xi)=X_{2}(1) X_{n, 1}(\xi)-X_{1}(1) X_{n, 2}(\xi) .
$$

The eigenvalues $\lambda_{n}$ and the associated eigenfunctions $\phi_{n}(\xi)$ are obtained from (51) and (52) by numerical analysis. Two coefficients in (28)-(30) are derived as

$$
\begin{gathered}
\gamma_{n}(\tau)=\bar{\gamma}_{n 1}(\xi) e^{-C_{0} \tau}+\bar{\gamma}_{n 2}(\xi), \\
B_{n}(\tau)=\left[\alpha_{n}+\frac{\bar{\gamma}_{n 1}(\xi)}{\lambda_{n}^{2}-C_{0}}+\frac{\bar{\gamma}_{n 2}(\xi)}{\lambda_{n}^{2}}\right] e^{-\lambda_{n}^{2} \tau} \\
-\frac{\bar{\gamma}_{n 1}(\xi)}{\lambda_{n}^{2}-C_{0}} e^{-C_{0} \tau}-\frac{\bar{\gamma}_{n 2}(\xi)}{\lambda_{n}^{2}},
\end{gathered}
$$

where

$$
\begin{gathered}
\delta_{n}=\left[\int_{\bar{r}}^{1} c_{m} \xi^{\beta_{2}+1} \phi_{n}^{2}(\xi) d \xi\right], \\
\bar{\gamma}_{n 1}(\xi)=\frac{\bar{\theta}_{2}}{\delta_{n}}\left\{\int _ { \overline { r } } ^ { 1 } \phi _ { n } ( \xi ) \left[c_{m} \xi^{\beta_{2}} C_{0}\left(a^{*}+b^{*} \xi\right)\right.\right. \\
\left.\left.+b^{*}\left(\beta_{1}+1\right) k_{m} \xi^{\beta_{1}-1}\right] d \xi\right\}, \\
\bar{\gamma}_{n 2}(\xi)=\frac{-b^{*} \bar{\theta}_{2}}{\delta_{n}}\left[\int_{\bar{r}}^{1} \phi_{n}(\xi)\left(\beta_{1}+1\right) k_{m} \xi^{\beta_{1}-1} d \xi\right], \\
\alpha_{n}=0 .
\end{gathered}
$$


TABLE 1: Temperature distribution of FG circular hollow cylinders with constant value of $\beta_{2}$ and various parameters of $\beta_{1}$ and $C_{0}$ at $\tau=0.5$, $\left[k_{m}=1, c_{m}=1, \theta(1, \tau)=\left(C_{0} \sin \omega \tau\right) \bar{\theta}_{2}: \bar{\theta}_{2}=4\right.$ and $\left.\omega=2.5\right]$.

\begin{tabular}{|c|c|c|c|c|c|c|c|c|}
\hline \multirow{2}{*}{$C_{0}$} & \multirow{2}{*}{$\beta_{1}$} & \multirow{2}{*}{$\beta_{2}$} & \multicolumn{6}{|c|}{$\xi$} \\
\hline & & & 0.5 & 0.6 & 0.7 & 0.8 & 0.9 & 1.0 \\
\hline \multirow{3}{*}{0.1} & 0.75 & \multirow{3}{*}{1} & 0 & 4.595 & 2.259 & 1.845 & 1.632 & 0.380 \\
\hline & 1 & & 0 & 4.592 & 2.285 & 1.990 & 1.820 & 0.380 \\
\hline & 1.25 & & 0 & 4.486 & 2.279 & 2.133 & 1.975 & 0.380 \\
\hline \multirow{3}{*}{1} & 0.75 & \multirow{3}{*}{1} & 0 & 45.952 & 22.589 & 18.453 & 16.320 & 3.796 \\
\hline & 1 & & 0 & 45.920 & 22.848 & 19.895 & 18.202 & 3.796 \\
\hline & 1.25 & & 0 & 44.865 & 22.788 & 21.329 & 19.752 & 3.796 \\
\hline \multirow{3}{*}{10} & 0.75 & \multirow{3}{*}{1} & 0 & 459.520 & 225.888 & 184.526 & 163.201 & 37.959 \\
\hline & 1 & & 0 & 459.196 & 228.480 & 198.950 & 182.017 & 37.959 \\
\hline & 1.25 & & 0 & 448.647 & 227.881 & 213.290 & 197.520 & 37.959 \\
\hline
\end{tabular}

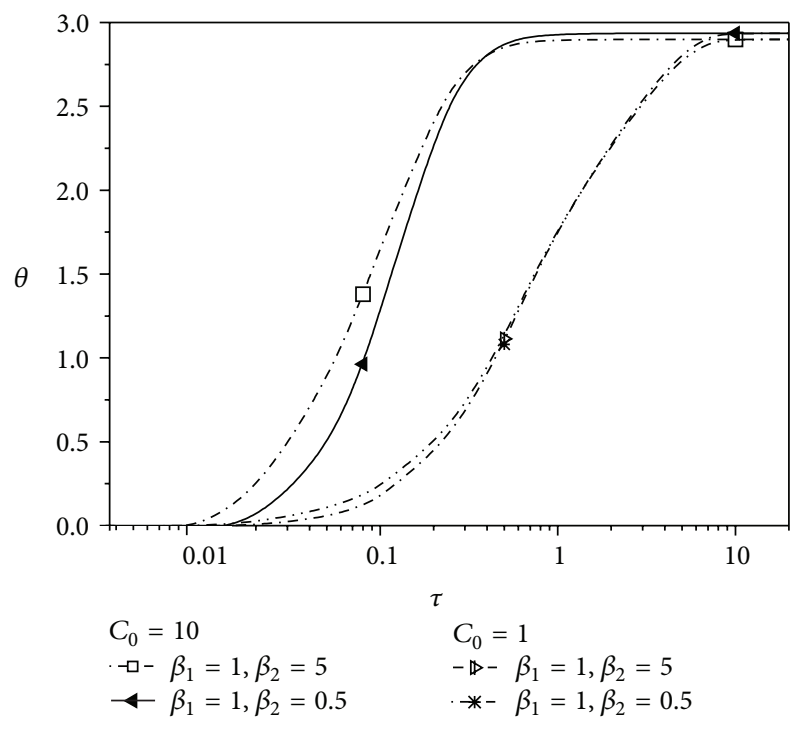

FIgURE 2: Temperature variation of FG circular hollow cylinders with constant value of $\beta_{1}$ and various values of $\beta_{2}$ and $C_{0}$ at $\xi=0.75$, $\left[k_{m}=1, c_{m}=1, \theta(1, \tau)=\left(1-e^{-C_{0} \tau}\right) \bar{\theta}_{2}: \bar{\theta}_{2}=4\right]$.

Consequently, the analytic closed solution for the system can be derived as

$$
\begin{aligned}
\theta(\xi, \tau) & \\
= & \frac{\left(1-e^{-C_{0} \tau}\right) \bar{\theta}_{2}}{1-\bar{r}}(\xi-\bar{r}) \\
+ & \sum_{n=1}^{\infty}\left\{X_{2}(1) X_{n, 1}(\xi)-X_{1}(1) X_{n, 2}(\xi)\right\} \\
& \times\left\{\begin{array}{l}
{\left[\frac{\bar{\gamma}_{n 1}(\xi)}{\lambda_{n}^{2}-C_{0}}+\frac{\bar{\gamma}_{n 2}(\xi)}{\lambda_{n}^{2}}\right] e^{-\lambda_{n}^{2} \tau}} \\
-\frac{\bar{\gamma}_{n 1}(\xi)}{\lambda_{n}^{2}-C_{0}} e^{-c_{0} \tau}-\frac{\bar{\gamma}_{n 2}(\xi)}{\lambda_{n}^{2}}
\end{array}\right\} .
\end{aligned}
$$

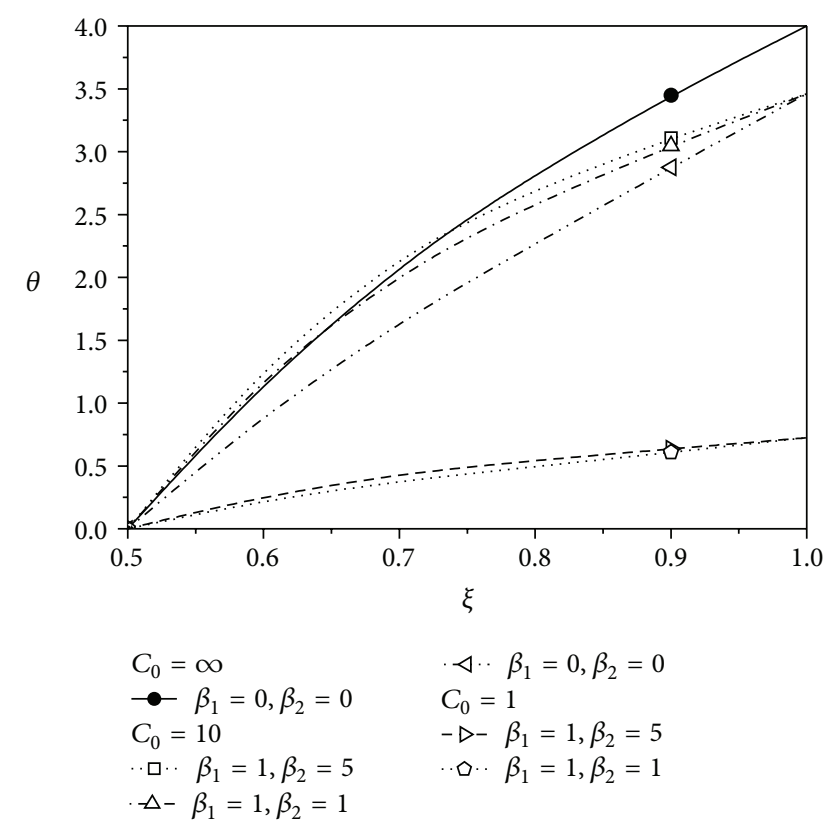

FIGURE 3: Temperature distribution of FG circular hollow cylinders with constant value of $\beta_{1}$ and various values of $\beta_{2}$ and $C_{0}$ at $\tau=0.2$, $\left[k_{m}=1, c_{m}=1, \theta(1, \tau)=\left(1-e^{-C_{0} \tau}\right) \bar{\theta}_{2}: \bar{\theta}_{2}=4\right]$.

In Figure 2, the temperature variation of FG circular hollow cylinders with various parameters of $\beta_{1}, \beta_{2}$, and $C_{0}$ at $\xi=0.75$ is shown. It can be found that when $C_{0}$ is a positive constant, the temperature parameter of the mediums at $\xi=0.75$ increases then reaches the associated constant temperatures over time. The temperature increase rate for the system with a higher value of $C_{0}$ is greater than that of one with a lower value of $C_{0}$. Figures 3 and 4 show the temperature distribution of FG circular hollow cylinders with various parameters of $\beta_{1}, \beta_{2}$, and $C_{0}$ at $\tau=0.2$. From these figures, it can be observed that with constant parameter $\beta_{1}$, the temperature of the mediums increases as parameter $\beta_{2}$ 


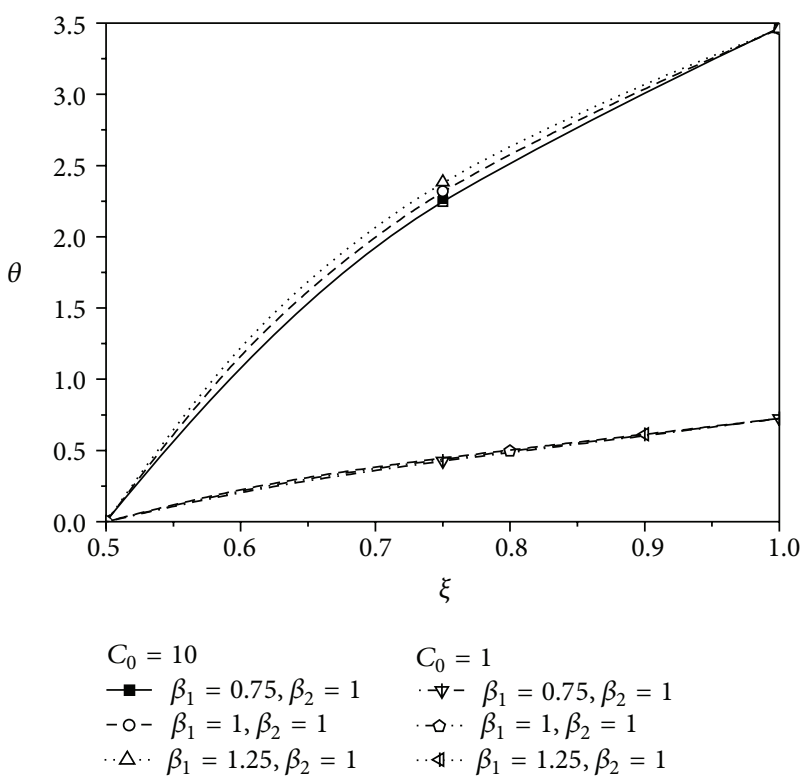

FIGURE 4: Temperature distribution of FG circular hollow cylinders with constant value of $\beta_{2}$ and various values of $\beta_{1}$ and $C_{0}$ at $\tau=0.2$, $\left[k_{m}=1, c_{m}=1, \theta(1, \tau)=\left(1-e^{-C_{0} \tau}\right) \bar{\theta}_{2}: \bar{\theta}_{2}=4\right]$.

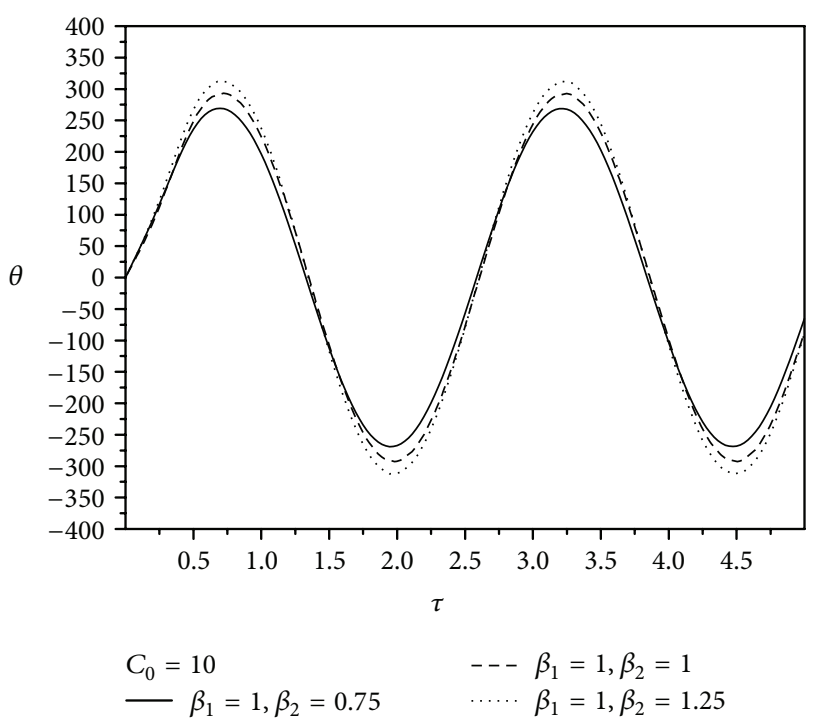

FIgURE 5: Temperature variation of FG circular hollow cylinders with constant value of $\beta_{1}$ and various values of $\beta_{2}$ at $\xi=0.75$, $\left[k_{m}=1, c_{m}=1, \theta(1, \tau)=\left(C_{0} \sin \omega \tau\right) \bar{\theta}_{2}: \bar{\theta}_{2}=4, C_{0}=10\right.$ and $\omega=2.5]$.

is increased. With constant parameter $\beta_{2}$, the temperature of the mediums increases as parameter $\beta_{1}$ is increased.

Example 3. Consider the same physical system as discussed in Example 2. In this case, the time dependent boundary condition at $\xi=1,(44)$, is changed to the form

$$
\theta(1, \tau)=\left(C_{0} \sin \omega \tau\right) \bar{\theta}_{2}
$$

In this case, the eigenvalues and eigenfunctions are the same as those given in Example 2.

Now,

$$
\begin{aligned}
F(\xi, \tau)=\frac{-\bar{\theta}_{2} C_{0}}{1-\bar{r}}[ & \left(\beta_{1}+1\right) k_{m} \xi^{\beta_{1}} \sin \omega \tau \\
& \left.-(\xi-\bar{r}) \omega c_{m} \xi^{\beta_{2}} \cos \omega \tau\right] .
\end{aligned}
$$

Following the same solution procedures as shown, the exact solution for the general system can be derived as

$$
\begin{aligned}
\theta(\xi, \tau)= & \frac{C_{0} \sin \omega \tau}{1-\bar{r}} \bar{\theta}_{2}(\xi-\bar{r}) \\
& +\sum_{n=1}^{\infty}\left[X_{2}(1) X_{n, 1}(\xi)-X_{1}(1) X_{n, 2}(\xi)\right] B_{n}(\tau),
\end{aligned}
$$

where

$$
\begin{aligned}
& B_{n}(\tau)= \frac{1}{\omega\left[\left(\lambda_{n}^{2} / \omega\right)^{2}+1\right]} \\
& \times\left\{\begin{array}{l}
\left(\frac{\lambda_{n}^{2}}{\omega} \cos \omega \tau+\sin \omega \tau-\frac{\lambda_{n}^{2}}{\omega} e^{-\lambda_{n}^{2} \tau}\right) \bar{\gamma}_{n 1}(\xi) \\
-\left(\frac{\lambda_{n}^{2}}{\omega} \sin \omega \tau-\cos \omega \tau-e^{-\lambda_{n}^{2} \tau}\right) \bar{\gamma}_{n 2}(\xi)
\end{array}\right\}, \\
& \bar{\gamma}_{n 1}(\xi)=\frac{-\bar{\theta}_{2} C_{0}}{(1-\bar{r}) \delta_{n}}\left[\int_{0}^{1} \phi_{n} \phi_{n}(\xi)(\xi-\bar{r}) \omega c_{m} \xi^{\beta_{2}+1} d \xi\right] \\
& \bar{\gamma}_{n 2}(\xi)=\frac{\left.-\bar{\theta}_{2} C_{0}(\xi) d \xi\right]}{(1-\bar{r}) \delta_{n}}\left[\int_{0}^{1} \phi_{n}(\xi)\left(\beta_{1}+1\right) k_{m} \xi^{\beta_{1}+1} d \xi\right], \\
& \alpha_{n}=0 .
\end{aligned}
$$

Figure 5 shows the harmonic temperature variation of FG circular hollow cylinders with constant value of $\beta_{1}$, various parameters of $\beta_{2}$ at $\xi=0.75, C_{0}=10$, and $\omega=2.5$. It can be observed that with constant value of $\beta_{1}$, the amplitude of temperature oscillation for the system with a higher value of $\beta_{2}$ will be more than that of one with a lower value of $\beta_{2}$.

In Table 1 , the temperature variations of FG circular hollow cylinders with constant value of $\beta_{2}$ and various values of $\beta_{1}$ and $C_{0}$ at $\tau=0.5$ are given. It can be observed that with constant parameter $\beta_{2}$, the temperature of the mediums will decrease as parameter $\beta_{1}$ is decreased.

\section{Conclusions}

The problem of heat conduction with general time-dependent boundary conditions cannot be solved directly by the method of separation of variables. In most of the analyses, an integral transform was used to remove the time-dependent term. 
In this paper, a new analytic solution method is developed to find the analytic closed solutions for the transient heat conduction in FG circular hollow cylinders with general time-dependent boundary conditions. The developed solution method is free of any kind of integral transformation.

By introducing suitable shifting functions, the governing second-order regular singular differential equation with variable coefficients and time-dependent boundary conditions is transformed into a differential equation with homogenous boundary conditions. The analytic solution of the system with thermal conductivity and specific heat in power functions with different orders is developed. Finally, limiting studies and numerical analyses are given to illustrate the efficiency and the accuracy of the analysis. The proposed solution method can also be extended to the problems with various kinds of FG materials and time-dependent boundary conditions.

\section{Acknowledgment}

It is gratefully acknowledged that this research was supported by the National Science Council of Taiwan, Taiwan, under Grant NSC 99-2221-E-006-021.

\section{References}

[1] M. N. Özisik, Boundary Value Problems of Heat Conduction, International Textbook Company, Scranton, $\mathrm{Pa}$, USA, 1st edition, 1968.

[2] Y. Obata and N. Noda, "Steady thermal stresses in a hollow circular cylinder and a hollow sphere of a functionally gradient material," Journal of Thermal Stresses, vol. 17, no. 3, pp. 471-487, 1994.

[3] H. Awaji and R. Sivakumar, "Temperature and stress distributions in a hollow cylinder of functionally graded material: the case of temperature-independent material properties," Journal of the American Ceramic Society, vol. 84, no. 5, pp. 1059-1065, 2001.

[4] G. N. Praveen and J. N. Reddy, "Nonlinear transient thermoelastic analysis of functionally graded ceramic-metal plates," International Journal of Solids and Structures, vol. 35, no. 33, pp. 4457-4476, 1998.

[5] M. Jabbari, S. Sohrabpour, and M. R. Eslami, "Mechanical and thermal stresses in a functionally graded hollow cylinder due to radially symmetric loads," International Journal of Pressure Vessels and Piping, vol. 79, no. 7, pp. 493-497, 2002.

[6] M. Jabbari, S. Sohrabpour, and M. R. Eslami, "General solution for mechanical and thermal stresses in a functionally graded hollow cylinder due to nonaxisymmetric steady-state loads," ASME Transactions Journal of Applied Mechanics, vol. 70, no. 1, pp. 111-118, 2003.

[7] Y. Ootao and Y. Tanigawa, "Transient thermoelastic analysis for a functionally graded hollow cylinder," Journal of Thermal Stresses, vol. 29, no. 11, pp. 1031-1046, 2006.

[8] J. Zhao, X. Ai, Y. Li, and Y. Zhou, "Thermal shock resistance of functionally gradient solid cylinders," Materials Science and Engineering A, vol. 418, no. 1-2, pp. 99-110, 2006.

[9] S. M. Hosseini, M. Akhlaghi, and M. Shakeri, "Transient heat conduction in functionally graded thick hollow cylinders by analytical method," Heat and Mass Transfer, vol. 43, no. 7, pp. 669-675, 2007.

[10] Y. Ootao, T. Akai, and Y. Tanigawa, "Three-dimensional transient thermal stress analysis of a nonhomogeneous hollow circular cylinder due to a moving heat source in the axial direction," Journal of Thermal Stresses, vol. 18, no. 5, pp. 497-512, 1995.

[11] Z.S. Shao and G. W. Ma, "Thermo-mechanical stresses in functionally graded circular hollow cylinder with linearly increasing boundary temperature," Composite Structures, vol. 83, no. 3, pp. 259-265, 2008.

[12] M. Jabbari, A. H. Mohazzab, and A. Bahtui, "One-dimensional moving heat source in a hollow FGM cylinder," ASME Transactions Journal of Pressure Vessel Technology, vol. 131, no. 2, article 021202, 7 pages, 2009.

[13] M. Asgari and M. Akhlaghi, "Transient thermal stresses in twodimensional functionally graded thick hollow cylinder with finite length," Archive of Applied Mechanics, vol. 80, no. 4, pp. 353-376, 2010.

[14] S. Singh, P. K. Jain, and R.-U. Rizwan-Uddin, "Finite integral transform method to solve asymmetric heat conduction in a multilayer annulus with time-dependent boundary conditions," Nuclear Engineering and Design, vol. 241, no. 1, pp. 144-154, 2011.

[15] P. Malekzadeh and Y. Heydarpour, "Response of functionally graded cylindrical shells under moving thermo-mechanical loads," Thin-Walled Structures, vol. 58, pp. 51-66, 2012.

[16] H. M. Wang and C. B. Liu, "Analytical solution of twodimensional transient heat conduction in fiber-reinforced cylindrical composites," International Journal of Thermal Sciences, vol. 69, pp. 43-52, 2013.

[17] S. Y. Lee and S. M. Lin, "Dynamic analysis of nonuniform beams with time-dependent elastic boundary conditions," ASME Transactions Journal of Applied Mechanics, vol. 63, no. 2, pp. 474-478, 1996.

[18] H. T. Chen, S. L. Sun, H. C. Huang, and S. Y. Lee, "Analytic closed solution for the heat conduction with time dependent heat convection coefficient at one boundary," Computer Modeling in Engineering and Sciences, vol. 59, no. 2, pp. 107-126, 2010. 


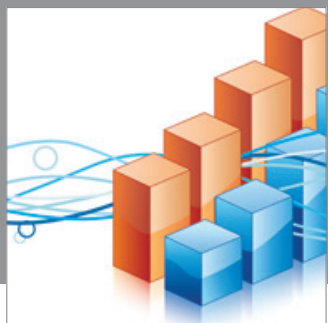

Advances in

Operations Research

mansans

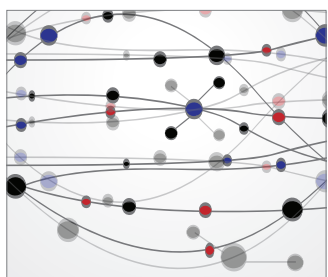

The Scientific World Journal
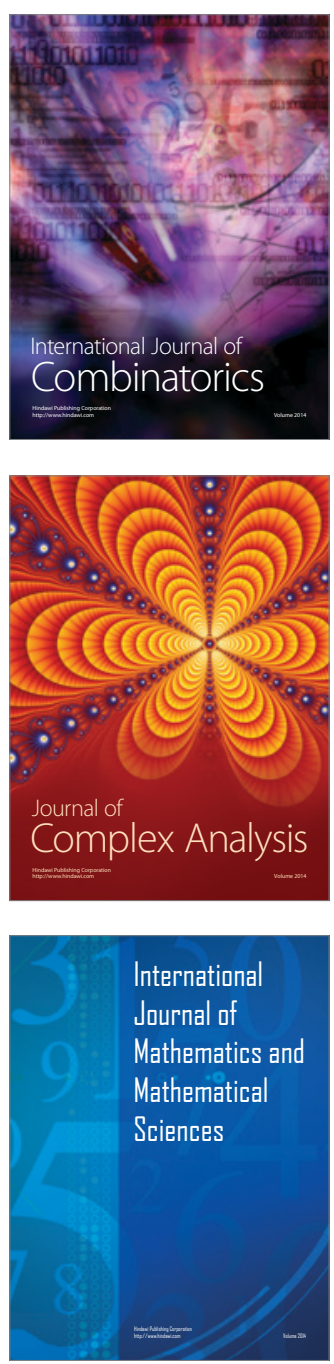
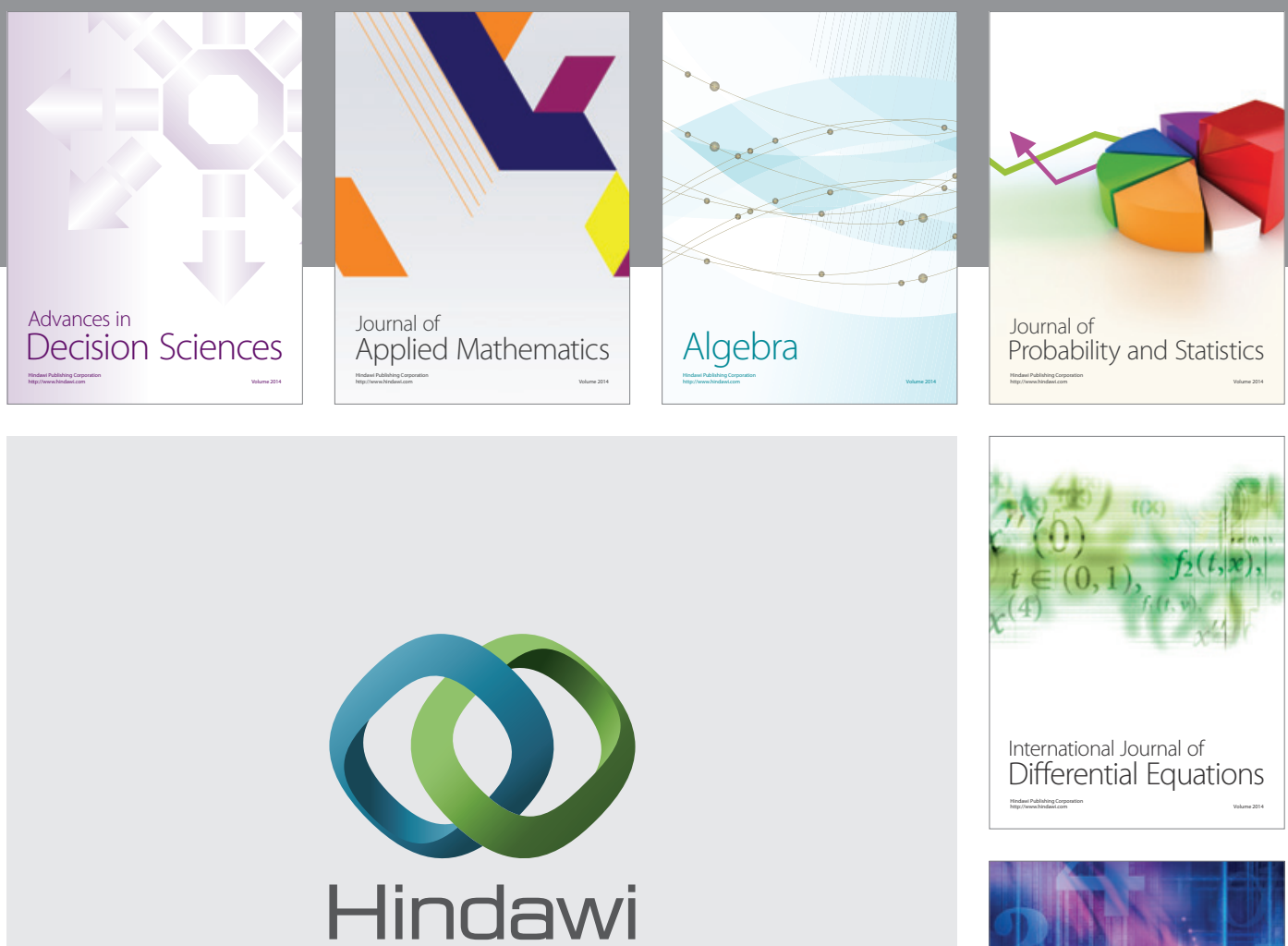

Submit your manuscripts at http://www.hindawi.com
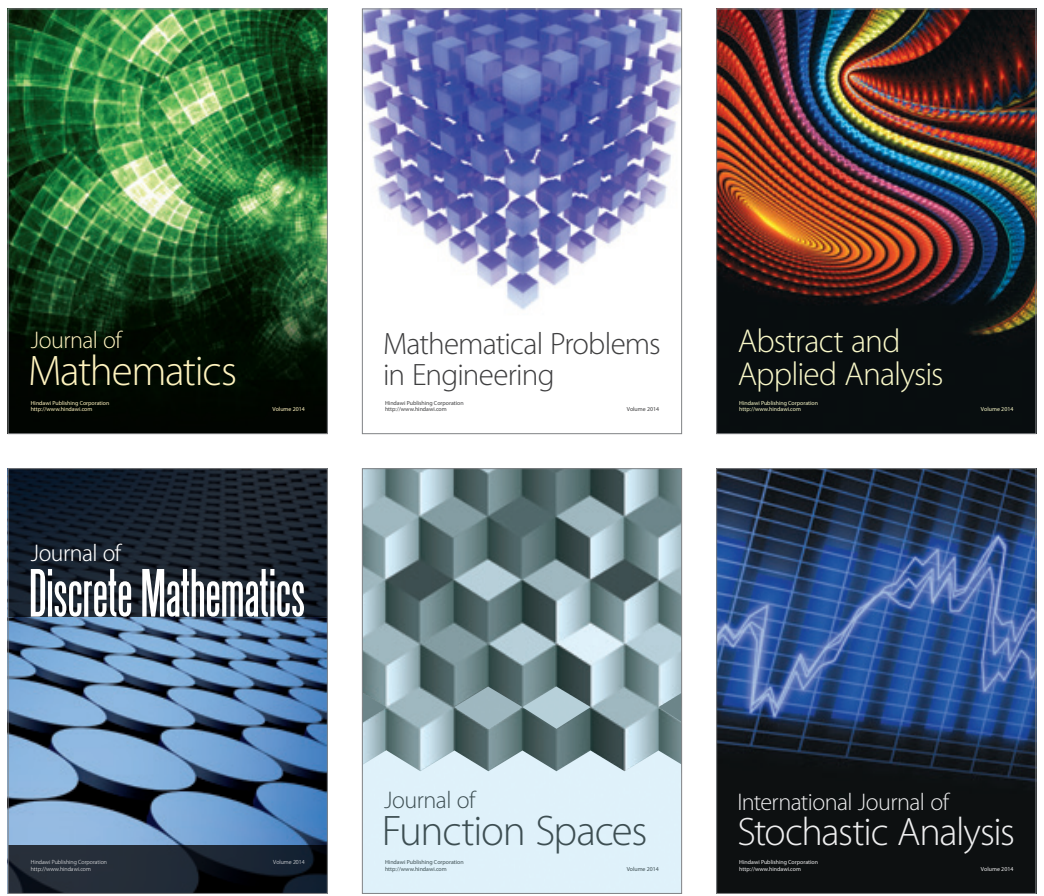

Journal of

Function Spaces

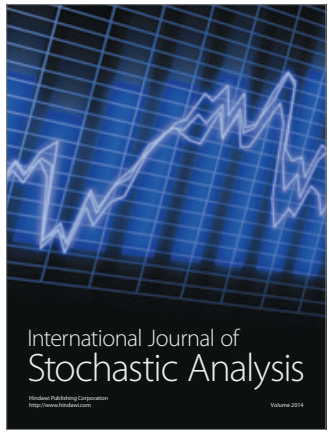

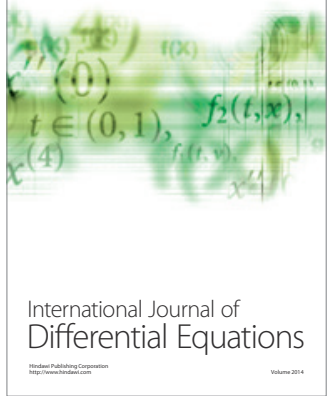
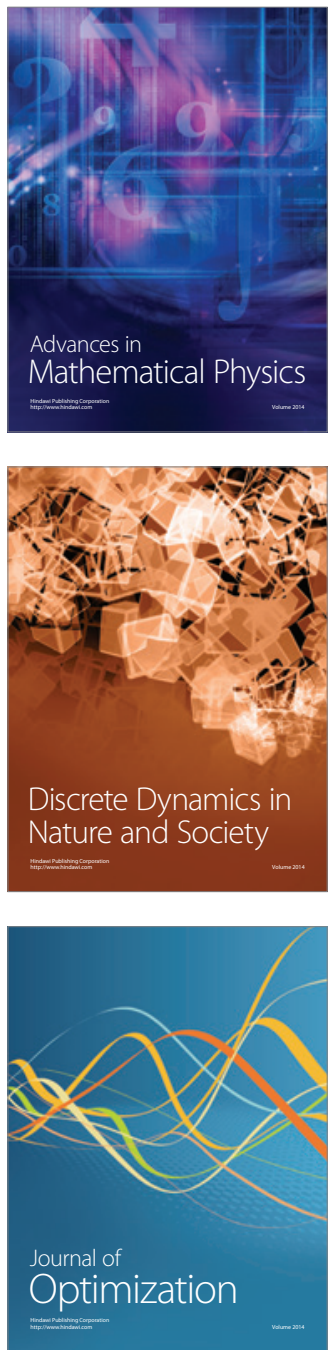